\title{
Un programme pour aider les étudiants en médecine à développer leurs stratégies d'apprentissage
}

\author{
Daniel J. CÔtÉ*, Colette B日 LAVANCE*, Martine CHAMBERLAND*, Ann GRAILLON*
}

\begin{abstract}
Résumé Buts: Exposer les considérations qui ont conduit à la création du programme d'enseignement des stratégies d'apprenti ssage aux éudiants de la faculté de médecine de I'U niversité de Sherbrooke; décrire ce programme. Contexte et problématique : Soucieuse d'apporter à ses étudiants une assistance pour la construction des compétences utiles à leurs fonctions professionnelles, cette institution réforme continuellement son curriculum en se basant sur les développements récents de la rechercheen pédagogie. Ces travaux mettent l'accent sur le processus d'apprentissage et justifient l'intérêt tant théorique qu'empirique de recourir à des stratégies d'apprentissage efficaces. En fait, cet intérêt prévaudrait aussi chez les étudiants en médecine. D escription de l'innovation : Ce programme d'enseignement des stratégies d'apprenti ssage est basé sur les conditions que la littérature indique comme propi cesà en favoriser le succès. II comporte cinq interventions au format dynamique et interactif, qui sétalent sur les quatre ans du cursus. L'ensemble du corps professoral est mis à contribution. Un large répertoi re de stratégies d'apprenti ssage utiles en médecine y est enseigné Ces stratégies sont apprises dans le contexte même des études médicales, en insisant particulièrement sur leur utilisation méacognitive. La production individuele de cartes conceptuel les joue un rôle majeur dans ce programme et suscite une forte motivation chez les étudiants. Conclusi on : Des mesures de l'impact de ce programme ne font que commencer. Il est toutefois permis d'en espérer, si l'on sen rapporte aux éudes publiées, des répercussions majeures sur la motivation des étudiants
\end{abstract}

M ots Clés A pprentissage; stratégies ; stratégies d'apprentissage ; stratégies d'étude; étudiants en médecine; enseignement ; programme.

Abstract G oals: To explain the rationale leading to the development of the Learning StrategiesTeaching Program deve loped at the Faculté de M édecine de l'U niversité de Sherbrooke; to describe this program. Context and problematic: To help its students construct useful competencies for their professional careers, this medical school continually revises its curriculum in light of any new development within the field of pedagogical research. This research has emphasized the importance of the learning process and has shown promise, on both theoretical and empi rical grounds, for effective learning strategies These strategies could benefit medical students as well. D escription of the innovation: This Learning Strategies Teaching Program is based upon conditions that the pedagogical literature favours for success. The four year program comprises five educational events, designed to be both dynamic and interactive. The entire faculty staff is involved and a large set of strategies useful in learning medicine is taught. These strategies are learned within the context of medicine with particular emphasis put on the metacognitive knowledge required to usethem. The construction of concept maps plays a major role in the program and this learning technique is a great motivator for the students. Conclusion: Evaluating the results of thisprogram has only just begun. H owever, in light of information available from published studies, it is expected to have a major impact on the motivation of students

Key Words Learning ; strategies ; learning strategies; study strategies; learning/study skills; medical students; teaching ; program.

Pédagogie M édicale 2004 ; 5. : 95-102

*Faculté de Médecine, Université de Sherbrooke, Québec, Canada

Correspondance : Daniel J. Côté, MD, M.Ed. - Faculté de Médecine del'Université de Sherbrooke - 3001, 12e avenue nord - J1H 5N4 SHERBROOKE, QC, Canada - Tel. : (819) 346-1110 poste 14403 - mailto:Daniel.J.Cote@USherbrooke.ca 


\section{Concepts et Innovations}

\section{Introduction}

Les étudiants en médecine ont-ils besoin d'un enseigne ment formel pour développer de meilleures stratégies d'apprentissage ? Si oui, quelles seraient ces stratégies et quelles conditions favoriserai ent le succès d'un programme dédié à cette intention ? Après avoir situé le contexte dans lequel ces questions se sont posées à la faculté de médecine de l'U niversité de Sherbrooke (FM US), nous énoncerons nos éléments de réponse puis décrirons le programme d'enseignement des stratégies d'apprentissage que nous avons mis en place. $N$ ous présenterons ensuite certains indicateurs de l'état des stratégies d'apprentissage chez nos étudiants actuels et conclurons en abordant des perspectives d'évaluation des retombées du programme.

\section{Contexte}

En 1987, la FM US a tranformé son cursus prédoctoral en privilégiant I'apprentissage par problèmes (APP) commeméthode pédagogiquecentrale'. D ix ans plustard, cependant, la routine sinstallait au niveau de la méthode; on observait une baisse de la motivation des étudiants et une insatisfaction des professeurs par rapport à leur rôle qu'ils jugeaient passif. Pour corriger ces éléments, l'approche pédagogiquea été de nouveau actual isée en 1997 : les étapes de la méthode APP sont demeurées sensible ment les mêmes, mais les tâches à l'intérieur de ces étapes et les interventions spécifiques des étudiants et des tuteurs ont été profondément modifiées. En présence de tuteurs experts, actifs et stratégiques dans leurs interventions, l'emphase se fait dorénavant davantage sur la réactivation et la validation des connaissances antérieures, sur I'élaboration et l'organisation des connaissances nouvelles et sur la finalité des apprentissages, c'est-à-dire la compétence clinique. L'étude individuelle des étudiants est désormais balisée : les stratégies d'apprentissage réputées efficaces sont explicitées et la production individuelle pour chaque problème d'une carte conceptuelle, outil que nous décrivons plus loin, est exigée.

Pour implanter ceschangements importants, une préparation adéquate des acteurs principaux était essentielle. Simultanément à une formation professorale d'environ une journée par année, a été ajoutée, pour les étudiants, un programme d'enseignement des stratégies d'apprentissage (PESA), que nous décrivons dans cet article.

Une autre raison justifiait la mise en place du PESA. Bien que les étudiants admis en médecine aient un dossier académique excellent, nous observions qu'un nombre significatif d'entre eux faisaient état d'une certaine détresse psy- chologique face à l'exigence de réussite dans leurs études médicales. Des entrevues individuelles mettaient en évidence des stratégies déficientes, une mauvaise gestion du temps, une anxiété accrue et une démotivation.

\section{D éfinition et classification des stratégies d'apprentissage}

Inspirés par Weinstein etal. (p.123)², nous appelons «stratégies » les " pensées ou actions que l'on choisit et régule délibérément, en vue d'atteindre un but précis » (traduction). Une stratégie «d'apprentissage» aura donc pour but de faire mieux apprendre.

Pour $\mathrm{C}$ averly et $\mathrm{O}$ rlando $0^{3}$, quatre perspectives ont marqué notre compréhension de l'apprentissage tout au long du $X X{ }^{e}$ siècle, chacune d'elles mettant en valeur différentes stratégies d'apprentissage. Chacune de ces perspectives englobe mais aussi dépasse les perspectives précédentes : 1) l'apprentissage a d'abord été vu comme le produit d'une série de compartiments mnémoniques ayant une capacité limitée; en termes destratégies, il convenait donc de sélectionner les informations les plus pertinentes et de les répéter régulièrement ; 2) la seconde perspective a plutôt mis l'accent sur les processus de traitement de l'information, en renvoyant à la notion d'« apprentissageen profondeur » et en valorisant al ors des stratégies comme l'éla boration et l'organisation ; 3) par la suite, la théorie des schémas a continuéà promouvoir ces deux stratégies mais, en insistant sur le contexte et sur l'importance des connaissances antérieures, elle a introduit la notion d'« apprentissage significatif »; 4) plus récemment, enfin, on a insisté sur l'importance, pour l'apprenant, de tenir compte des conditions dans lesquelles chacune des straté gies d'apprentissage sapplique plus spécifiquement ; à titre d'exemple, une stratégie simple telle que la répétition est plus efficiente lorsqu'il sagit de réaliser un apprentissage routinier.

Parce qu'elle sappliquait bien aux compétences professionnelles visées par les éudes médicales, nous avons utilisé, comme classification des stratégies d'apprentissage, celle de Boulet et al. ${ }^{4}$ (tableau 1).

En médecine, le champ d'application privilégié des connaissances procédurales est celui des habiletés cliniques, c'est-à-dire celles requises pour l'anamnèse et l'exa men physique. La stratégie favorisant l'apprentissage de ce 


\section{Tableau 1: \\ Une classification des stratégies d'apprentissage \\ (d'après A. Boulet et al. ${ }^{4}$ et J.Tardifi' ${ }^{14}$ )}

\section{STRATÉGIES COGNITIVES}

\section{Apprentissage de connaissances déclaratives}

Répétition: $\quad$ Reformulation des informations dans les mêmes termes

Élaboration : $\quad$ Reformulation des informations dans des termes différents, en ajoutant

des informations personnelles à celles qui sont présentées

O rganisation : $\quad H$ iérarchisation, catégorisation et transformation des informations

\section{Apprentissage de connaissances procédurales}

Compilation: Décomposition des connaissances relatives à une activité complexe en séquences plus simples (procéduralisation) puis induction d'une représentation plus globale (composition)

\section{Apprentissage de connaissances conditionnelles}

Généralisation : $\quad$ Reconstruction de connaissances de manière à ce qu'elles sappliquent à plusieurs cas

Discrimination : Restriction du répertoire de situations auxquelles sappliquent des connaissances

\section{STRATÉGIES MÉTACOGNITIVES}

Planification : $\quad$ Ordonnancement de ses activités d'apprentissage

Contrôle : $\quad$ Jugement porté sur ses activités d'apprentissage en cours

Régulation: (Ré)ajustement de ses activités par rapport à ses buts

\section{STRATÉGIES AFFECTIVES}

Pour maintenir sa motivation

Pour maintenir sa concentration

Pour contrôler son anxiété

\section{STRATÉGIES DE GESTION}

Pour gérer son temps efficacement

Pour gérer les ressources matérielles (dont l'environnement d'étude)

Pour gérer le soutien de personnes

genre de connaissances est la compilation, qui consiste à décomposer des connaissances relatives à des activités complexes en séquences plus simples (procéduralisation), afin de pouvoir les interpréter plus facilement, puis à induire une représentation plus globale (composition), en comparant sa performance à celle d'un modèlet. Comme ces pratiques guidées se font déjà dans le cadre de l'unité d'enseignement des habiletés cliniques, nous n'avons pas cru nécessaire de revenir sur les connaissances procédurales au sein du PESA.

Selon $\mathrm{G}$ ombert ${ }^{5}$ (cité par Wolfs ${ }^{6}$, p. 28), la métacognition est le «domaine qui regroupe: 1) les connai ssances introspectives conscientes qu'un individu particulier a de ses propres états et processus cognitifs ; 2 ) les capacités que cet 


\section{Concepts et Innovations}

individu a de délibérément contrôler et planifier ses propres processus cognitifs en vue de la réalisation d'un but ou d'un objectif déterminé »En accord avec cette définition, Boulet et al. ${ }^{4}$ reconnaissent que les buts d'apprentissage font appel à des «stratégies métacognitives » qui consistent à planifier, contrôler et au besoin réguler sa façon d'apprendre.

\section{Fondements du programme d'enseignement des stratégies d'apprentissage (PESA)}

\section{Intérêt du PESA}

Pressley et al. ${ }^{7}$ ont revu en 1997 la littérature relative aux stratégies d'étude des étudiants de niveau post-secondaire. Ils en concluaient que " good information processing is anything but universal among students » (p. 5). Ils utilisent le mot « often » pour décrire la fréquence des déficiences rencontrées au niveau des stratégies d'élaboration, d'organisation et de méacognition. Peut-on en dire autant des étudiants en médecine?

Lahtinen et al. ${ }^{8}$ ont invité 487 postulants à la faculté de médecine d'H elsinki à étudier pendant une heure le contenu d'environ 17 pages portant sur des notions de statistiques et d'épidémiologie. Ils étaient informés qu'ils pouvai ent écrire à volonté sur les copies ainsi que sur les feuilles vierges mises à leur disposition et qu'ils devraient ensuite passer un test sur cette matière, sous forme de questions à choix multiples et d'essai. A la fin de la période d'étude, tout le matériel était ramassé et les sujets étaient soumis au test. II devenait donc possible d'analyser les stratégies que les candidats avaient spontanément mises en œuure. Seuls $27 \%$ des étudiants avaient eu recours à des stratégies réputées favoriser un apprentissage significatif, c'est-à-dire qu'ils avaient élaboré un résumé dans leurs propres mots ou encore organisé activement les nouvelles connaissances.

Cartier et al. ${ }^{9}$ ont publié en 2001 une étude réalisée de 1995 à 1997 (donc avant l'introduction du PESA) auprès de 20 étudiants de troisième année de notre propre faculté. A l'aide d'une approche qual itative, ils ont montré que ces étudiants recouraient peu aux stratégies d'élaboration et d'organisation. En termes de métacognition, ces étudiants avaient l'habitude d'évaluer (contrôler) leur façon d'étudier mais n'utilisaient pas les informations ainsi acquises pour réajuster (réguler) si nécessaire leurs choix stratégiques.

On peut, toutefois, penser que ce profil se modifie au moment où l'étudiant en médecine aborde la période des stages cliniques. A cet égard, Gruppen et al. ${ }^{10}$, de la M ichigan M edical School, ont étudié 107 deleurs stagiaires de troisième année, au moment du stage de trois mois en médecine interne. Ces étudiants devaient auto-évaluer, à l'entrée en stage, leur connaissance des 14 problèmes cliniques caractérisant les objectifs de ce stage. En fin de stage, ils devaient rapporter le temps qu'ils avai ent consacréà étudier chacun de ces problèmes. Aucune corrélation n'a été trouvée entre les auto-évaluations initiales et les temps d'étude. Les auteurs concluent que leurs étudiants en médecine étaient néophytes en regard des stratégies métacognitives.

Il semble donc qu'au niveau post secondaire en général et possiblement même au niveau universitaire en médecine -, tous les étudiants ne soient pas capables d'acquérir spontanément les stratégies propres à favoriser un apprentissage significatif. Un contexte éducatif global qui sollicite et favorise l'utilisation de stratégies efficaces est dès lors un choix attrayant. D ans le cadre d'un tel système, se doter d'un programme enseignant de telles stratégies est une hypothèse qui pourrait s'avérer utile.

\section{Conditions de succès des programmes d'ensignement des strategies d'apprentissage}

Une recension des écrits nous permet d'identifier sept conditions favorisant le succès de ces programmes : 1) convaincre les étudiants de la pertinence, pour euxmêmes, qu'ils développent leurs stratégies d'apprentissa$g^{2}$; 2) montrer comment appliquer ces stratégies, en offrant des occasions répétées de pratique guidée par un feed-back ; 3) enseigner non pas une seule tactique, mais plutôt un répertoire de stratégies dans lequel l'étudiant pourra puiser non seulement des stratégies cognitives mais aussi des stratégies métacognitives ${ }^{11}$, affectives ${ }^{12}$ et de ges tion $^{4}$; 4) développer aussi la connaissance des conditions dans lesquelles telle ou telle stratégie sapplique plus spécifiquement2,3,4; 5) enseigner les stratégies d'apprentissage dans le contexte disciplinaire même de l'étudiant ${ }^{11}$; 6) sassurer qu'un tel programme soit pleinement soutenu par le corps professoral ${ }^{12}$ et : 7) vérifier que le système d'évaluation soit congruent avec la philosophie du programme ${ }^{2}$.

Q uels résultats peut-on espérer d'un programme qui mettrait en œuvre l'ensemble des conditions favorisantes 
détaillées ci-dessus ? La méta-analyse faite par $\mathrm{H}$ attie et al. ${ }^{12}$ montre que de tels programmes : 1) améliorent modérément la performance académique des sujets qui y ont participé ; 2) améiorent de façon très importante des facteurs affectifs, en augmentant par exemple le sentiment d'être compétent pour apprendre, en stimulant la motivation face aux études et en réduisant l'anxiété de performance mais : 3) ont très peu d'effet sur la façon même d'étudier des participants. II est à noter que les étudiants sur lesquels porte cette conclusion étaient hétérogènes en termes de niveau scolaire, de réussite académique, de statut socioéconomique et de bien d'autres attributs.

\section{Description du programme d'enseignement des stratégies d'apprentissage à la faculté de médecine de l'U niversite de Sherbrooke}

Le PESA mis en œuvre à la FM US comporte cinq composantes (Tableau 2). Conjointement avec les autres éléments de notre système éducatif, il satisfait la plupart des conditions favorisantes précédemment décrites. D'abord, trois de ses cinq composantes ont lieu à un moment où le type d'apprentissage à effectuer change (connaissances déclaratives, connaissances conditionnelles, milieu clinique), ce qui facilite la démonstration de la pertinence d'acquérir de nouvelles stratégies. Les étudiants ont l'occasion de mettre en pratique les stratégies d'apprentissage et de recevoir un feed-back expert. Ils sont amenés à réflé chir aux conditions dans lesquelles telle ou telle stratégie sapplique préférentiellement. Le soutien professoral au PESA est manifeste. Toutes les composantes du programme sont contextualisées au contenu médical spécifique à apprendre lors de chaque séquence et adoptent le format suivant : les quelque 120 étudiants sont réunis par petits groupes, encadrés au total et selon les activités par 2 à 15 professeurs; I'atelier, qui dure de 3 à 8 heures, comprend des moments d'apports conceptuels sous forme magistrale, de réflexion individuelle, d'exercices en dyades, de tâches en petits groupes, de rapports en séance plénière. Les étudiants apportent leurs cahiers annotés, leurs résumés, etc... La participation à ces ateliers est obligatoire, créditée et publicisée par les autorités facultaires.

\section{Introduction aux stratégies d'apprentissage}

Cette première composante du PESA a lieu dès la deuxième semaine du cursus, c'est à dire entre le premier et le deuxième problème d'APP. Ce positionnement chronologique permet de partir des stratégies que les étudiants ont spontanément utilisées lors du premier problème, puis d'utiliser dès le deuxième problème les stratégies enseignées. Ces stratégies, présentées dans le tableau 3, sont propices à l'apprentissage des connaissances déclaratives,

\begin{tabular}{|c|c|c|c|c|c|c|c|c|}
\hline \multicolumn{9}{|c|}{$\begin{array}{l}\text { Tableau } 2 \text { : } \\
\text { Les cinq composantes du programme d'enseignement des stratégies d'apprentissage } \\
\text { (PESA) à la faculté de médecine de l'université de Sherbrooke }\end{array}$} \\
\hline & \multicolumn{2}{|c|}{$1^{\text {ère }}$ année } & \multicolumn{2}{|c|}{$2^{\text {ème }}$ année } & \multirow{2}{*}{\begin{tabular}{|c|}
$3^{\text {ème }}$ année \\
Août
\end{tabular}} & \multicolumn{3}{|c|}{ Externat } \\
\hline & Août & Janvier & Août & Janvier & & Janvier & Août & Janvier \\
\hline Introduction aux SA & $x$ & & & & & & & \\
\hline $\begin{array}{l}\text { Retours } \\
\text { en tutoriaux sur les SA }\end{array}$ & $X X X$ & $X X X$ & $X X X$ & $X X X$ & & & & \\
\hline Réflexion sur une année de SA & & & $x$ & & & & & \\
\hline SA appliquées au diagnostic & & & & & $x$ & & & \\
\hline SA en milieu dinique & & & & & & $x$ & & \\
\hline
\end{tabular}




\section{Concepts et Innovations}

\begin{tabular}{|c|} 
Tableau 3 : \\
Les stratégies d'apprentissage \\
enseignées lors de la composante \\
« Introduction aux stratégies \\
d'apprentissage », dans le cadre \\
du programme d'enseignement \\
des stratégies d'apprentissage (PESA) \\
- Gérer son temps \\
\hline - M aintenir sa motivation \\
- Gérer les ressources matérielles \\
que sont les références \\
- Élaborer \\
\hline - O rganiser en une carte conceptuelle \\
\hline - Contrôler, au besoin réguler, \\
ses stratégies d'apprentissage \\
\hline - Répéter (réviser) de temps à autre
\end{tabular}

type de connaissances principalement visées durant les deux premières années du cursus. Les stratégies favorisant un apprentissage davantage significatif (c'est-à-dire celles consistant à élaborer et à organiser) y sont priorisées.

Élaborer, c'est ajouter aux nouvelles informations des informations qui viennent de soi. Ainsi : reformuler/résumer dans ses propres mots (à voix haute ou au moment de prendre des notes), annoter dans les marges, paraphraser, formuler des questions, imaginer des analogies ou des exemples d'applications cliniques.

O rganiser, c'est catégoriser et hiérarchiser les nouvelles informations, en créant des liens conceptuels entre elles et avec les connaissances antérieures, que ce soit en dressant un plan, en construisant une synthèse ou encore une « carte conceptuelle ».

La carte conceptuelle est un moyen de représenter graphiquement, de façon visuelle et propositionnelle, l'organisation de ses connaissances : il sagit, par exemple en une seule page, d'illustrer les principaux concepts appris, ainsi que les relations entre ces concepts $s^{13,14,15}$.

Bien que les étudiants, lors de cet atelier, prennent conscience de la nécessité de contrôler et de réguler leurs choix stratégiques en cas de difficulté de compréhension, d'attention ou de motivation, on ne va guère plus loin dans I'utilisation métacognitive des stratégies. Ceci sera la préoccupation des composantes « retours en tutoriaux » et " réflexion sur une année d'utilisation des stratégies d'apprentissage ».

\section{Retours en tutoriaux sur les stratégies d'apprentissage}

Ces « retours » se font tout au long des unités d'APP et non dans un atelier distinct. Ils comportent deux moments : le feed-back sur les cartes conceptuelles, l'échange sur les stratégies. Pour renforcer la stratégie d'organisation des connai ssances, les tuteurs donnent un feedback systématique sur les cartes conceptuelles produites par les étudiants. C efed-back est écrit et individuel.

A deux reprises lors de chaque unité d'APP, les tuteurs animent un échange d'une quinzaine de minutes sur les stra tégies mises en œuvre par les étudiants lors de l'étude du problème. Les tuteurs évitent toute prescription et recourent plutôt à des questions ouvertes comme : «En ce début d'unité, comment différenciez-vous l'essentiel de l'accessoire?», «En quoi cette nouvelleunitévous amènet-elle à modifier des éléments de votre façon d'étudier? », "Que faites-vous quand vous ne comprenez pas? ", "Comment savez-vous que vous avez atteint vos objectifs? », etc...

Pour remplir ces fonctions, nos quelque deux cent méde cins professeurs ne sont nullement des experts en psychologie cognitive mais ils sont préparés par la formation professorale mentionnée précédemment.

\section{Réflexion sur une année d'utilisation des stratéges d'apprentissage}

Cet atelier a lieu au début de la deuxième année $Y$ sont ciblées les conditions dans lesquelles telle ou telle stratégie sapplique préférentiellement.

En effet, selon plusieurs auteurs' 4, 16, l'étudiant métacognitif planifie (et au besoin réajuste) ses stratégies en fonction de : 1) ce qu'il sait de lui-même comme apprenant (ses buts, ses préférences, ses connaissances antérieures) ; 2) ce qu'il perçoit de la tâche à accomplir (complexité, étapes à parcourir, temps disponible) ; 3) la nature du matériel à étudier (Iongueur, niveau de difficulté, structureexplicite ou implicite) et : 4) des exigences du professeur ou du type d'examen à venir. II apparaît ainsi que : 1) les stratégies d'élaboration et d'organisation sont plus effi- 
cientes quand on vise l'apprentissage significatif d'un matériel difficile en vue d'un essai ou d'une résolution de problèmes; 2) la stratégie de répétition (soulignement ou élaboration d'un résumé utilisant strictement le verbatim) convient mieux lorsqu'il s'agit de retenir les détails d'un matériel plus facile en vue d'un examen utilisant des questions à choix multiple; 3 ) dans tous les cas, la révision (de préférence périodique) est une condition nécessaire (quoique non suffisante) pour maintenir à niveau les connaissances nouvellement acquises

\section{Stratégies d'apprentissage appliquées au diagnostic}

La troisième année, d'août à décembre, a lieu l'unité multidisciplinaire Alors que les unités précédentes étaient unidisciplinaires (par exemple, cardiologie, santé mentale, etc.), cette unité vise l'intégration des connaissances, au service tout particulièrement du diagnostic médical. $\mathrm{Or}$, le diagnostic médical est le moment privilégié pour l'apprentissage des connai ssances conditionnelles.

L'accent est par conséquent mis sur l'apprentissage des stratégies propresà cetype de connaissances4, c'est-à-dire: 1) la généralisation (génération d'autres exemples de manifestation du diagnostic étudié, en montrant bien les ressemblances entre ces différents cas) et : 2) la discrimination (évocation d'au moins un cas qui, à première vue, ressemble au diagnostic étudié mais en est en fait un contre-exemple et argumentation de ce qui rend ces cas dissemblables).

\section{Stratégies d'apprentissage en milieu dinique}

Q uelques mois après le début de leur externat, les étudiants sont amenés à réfléchir à l'opportunité et aux moyens d'adapter les stratégies qu'ils ont utilisées dans le contexte des trois premières années (généralement livresque, étude personnelle en dehors de l'action) au nouveau contexte des stages (milieu clinique, apprentissage dans l'action centrée sur le patient).

De plus, comme les séances d'apprentissage au raisonnement clinique jouent un rôle important durant l'externat à Sherbrooke ${ }^{17}$, les étudiants discutent de la façon de " prolonger » ces séances au moment de leur étude personnelle.

\section{État actuel des stratégies d'apprentissage desétudiants de la faculté de médecine del'Université de Sherbrooke}

Les données qui suivent ne permettent pas une évaluation « causale » de l'impact éducationnel du PESA. Elles ne permettent pas d'isoler un élément particulier du système éducatif et résultent uniquement de la perception qu'ont les étudiants de leurs stratégies actuelles.

Avec ces limites, nous pouvons faire état des informations dont nous disposons.

Chaque composante du PESA se clôt par un sondage écrit. La majorité des étudiants jugent que le programme est pertinent et intéressant. Par ailleurs, chaque étudiant répond systématiquement depuis l'automne 1998 à un questionnaire d'évaluation de chacune des unités des deux premières années du cursus (quinze unités en tout). Certaines des questions portent sur l'étude personnelle. Une banque de plusieurs milliers de réponses est ainsi constituée G lobalement, les étudiants de première année rapportent utiliser " souvent » les stratégies enseignées, quoique de façon inégale (la génération d'exemples d'application est, notamment, significativement moins effective). Cette utilisation se maintient en deuxième année, moment où s'exprime par ailleurs une tendance à utiliser davantage les stratégies métacognitives. Les étudiants rapportent une grande motivation à créer leurs cartes conceptuelles.

Bédard et Viau ${ }^{18}$ ont conçu un questionnaire sur la façon d'apprendre des étudiants qui a été administré pendant l'automne 2000 à 4820 étudiants de l'U niversité de Sherbrooke, dont 223 de la FM US. O $n$ y rapporte queles étudiants en médecine ont confiance en leurs stratégies d'apprentissage et maintiennent une forte motivation à apprendre tout au long des quatre années du curriculum. Ils utilisent une variété de stratégies d'apprentissage, parmi lesquel les fréquemment l'élaboration ou l'organisation des connaissances.

Enfin, selon Chamberland et al. ${ }^{19}$, nos étudiants indiquent qu'ils perçoivent la construction de cartes conceptuelles comme un outil métacognitif important dans la structuration des connaissances.

\section{Conclusion}

D es recherches suggèrent l'intérêt des programmes d'enseignement de stratégies d'apprentissage. Nous avons 


\section{Un programme pour aider les étudiants en médecineà développer...}

donc construit, pour les étudiants en médecine, un programme longitudinal en cinq composantes, qui réunit les conditions réputées favoriser le succès de tels programmes. Parmi elles, la contextualisation, la considération des conditions dans lesquelles les stratégies sappliquent respectivement et le soutien professoral sont les principales. Les principales retombées d'un tel programme semblent concerner essentiellement la confiance des étudiants envers leur compétence d'apprenant et leur motivation à étudier. II conviendrait toutefois que nous modifiions nos façons d'évaluer les étudiants, de manièreà les rendre plus cohérentes avec la philosophie du programme.

\section{Remerciements}

N ous remercions particulièrement Monseur René Hivon, professeur titulaireà la facultéd'éducation de l'U niversité de Sherbrooke, pour ses conseils avisés au moment de la prélecture du manuscrit. N ous sommes toutefois seuls responsables dela version finale.

\section{Références}

1. Des $M$ archais JE, Bureau $M$, D umais $B$, Pigeon, $G$. From traditional to problem-based learning : A case report of complete curriculum reform. M ed Educ 1992; $26: 190-99$.

2. Weinstein CE, Goetz ET, Alexander PA. Learning and study strategies. Issues in assessment, instruction, and evaluation. San Diego, (CA) : Academic Press Inc., 1988.

3. Caverly DC, O rlando VP. Textbook study strategies In : RF Flippo and DC Caverly (Eds.), Teaching reading and study strategies at the college level. Newark : International Reading Association, 1991 : 85-165

4. Boulet A, SavoieZajc L, Chevrier J. Les stratégies d'apprenti ssage à l'université. Sainte-Foy $(\mathrm{Q} C)$ : Presses de I'U niversité du Q uébec, 1996.

5. Gombert JL. Le dével oppement métalinguistique. Paris: Presses U niversitai res de France, 1990.

6. Wolfs J-L. M éthodes de travail et stratégies d'apprentis sage Bruxelles: D e Boeck, 1998.

7. Pressey $M$, Yokoi $L$, van $M$ eter $P$, van Etten $S$, F reebern $G$. Some of the reasons why preparing for exams is so hard: What can be done to make it easier? Educ Psycho Rev $1997 ; 9: 1-38$.

8. Lahtinen V, L onka K, Lindblom-Ylänne S. Spontaneous study strategies and the quality of knowledge construction. Br J Educ Psychol $1997 ; 67$ : 13-24.

9. Cartier S, Plante A, Tardif J. Learning by Reading: D escription of L earning Strategies of Students I nvolved in a Problem-Based Learning Program. ERIC report no ED $452511 ; 2001$.

10. Gruppen LD, White C, Fitzgerald T, Grum CM , Woolliscroft JO. M edical student's self-assessments and their allocations of learning time. Acad M ed 2000 ; $75: 374-379$.
11. Simpson M L, N ist SL. An update on strategic learning : It's more than textbook reading strategies J Adolesc Adult Literacy $2000 ; 43$ : 528-541.

12. H attie J, Biggs J, Purdie N. Effects of learning skills interventions on student learning: A meta-analysis. Rev Educ Res 1996 ; 66 : 99-136.

13. N ovak JD, Gowin D B. Learning how to learn. N ew York : Cambridge U niversity Press 1984.

14. Tardif J. Pour un enseignement stratégique : L'apport de la psychologie cognitive. Montréal : Les Éditions Logiques 1992.

15. M archand C, Gagnayre R. Utilisation de la carte conceptuelle auprès de formateurs en santé pour l'apprentissage de concepts pédagogiques. Pédagogie M édicale $2004 ; 5$ : 13-23.

16. $\mathrm{H}$ adwin $A F$, Winne PH. Study strategies have meagre support: A review with recommendations for implementation. J Higher Educ 1996 ; 67 : 692-715.

17. Chamberland M. Les séances d'apprentissage du raisonnement clinique (ARC) : Un exemple d'activité pédagogi que contextualisée adaptée aux stages cliniques en médecine. Ann M éd Interne 1998 ; 149 : 479-484.

18. Bédard D, Viau R. Le profil d'apprentissage des étudiantes et des étudiants de I'U niversité de Sherbrooke. M anuscrit inédit, Sherbrooke (QC) : Université de Sherbrooke, 2001.

19. Chamberland M, Grand'M aison P, Plante A, H ivon R. Étude de cas sur l'utilisation du schéma dansl'APP. $M$ anuscrit inédit, Sherbrooke (QC) : Université de Sherbrooke, 1999. 